\section{P71 (continued)}

Objective: To improve dietary behavior of guardians of adolescents participating in a lifestyle weight management program.

Target Audience: Guardians of overweight and obese Hispanic adolescents participating in the Confidence, Ownership, Responsibility, and Exercise (CORE) lifestyle weight management program.

Theory, Prior Research, Rationale: The CORE lifestyle program was designed to improve body composition and weight management behaviors among overweight and obese Hispanic adolescents. As research suggests that effective obesity treatment in adolescents requires involvement of the family, an educational program was developed to teach guardians of CORE participants about nutrition for health promotion and weight management and improve dietary behavior. Curriculum was based on Social Cognitive Theory, the Dietary Guidelines for Americans, and American Academy of Pediatrics and Academy of Nutrition and Dietetics position papers on pediatric weight management.

Description: Nutrition education consisted of one, 60minute session per week over 12 weeks. Lessons were taught in both Spanish and English by a registered dietitian or trained nutrition intern and volunteer translator. All sessions were interactive and included hands on activities. Topics included MyPlate, sugary beverages, choosing healthy fats, and reading nutrition labels.

Evaluation: Following the program, guardians completed a retrospective, researcher-designed questionnaire to assess likelihood of engaging in healthy dietary behaviors before and after the program. Wilcoxon signed-rank tests were used to compare pre and post data. Twenty-six guardians provided data (mean age $38.8 \pm 5.7 \mathrm{y}, 70.4 \%$ female, $59.2 \% \leq$ middle-school education). Mean \pm SD attendance was $56.7 \pm 0.3 \%$ of sessions. Guardians reported increased likelihood of choosing water instead of sugary drinks, using nutrition labels, choosing whole grains, choosing vegetables in place of higher calorie foods, and choosing low fat/fat free milk and dairy (water: $P<.05$, all other behaviors: $P<.01)$.

Conclusions and Implications: The CORE nutrition education program was successful in increasing likelihood of engaging in healthy dietary behaviors among guardians of overweight and obese Hispanic adolescents. Future studies should address barriers to attendance in this population.

Funding: Jackson EMC Foundation, Mulherin Foundation.

\section{P72 Investigating Dietary and Physical Activity Patterns Among Hmong American Youth: A Cultural Perspective}

Urvashi Mulasi,PhD, MS, RDN, mulasi@csus.edu, California State University, Sacramento, 6000 J Street, Sacramento, CA 95819; Lisa Franzen-Castle, PhD, MS, RDN, University of Nebraska-Lincoln; Mical Kay Shilts, PhD, California State University, Sacramento
Background (Background, Rationale, Prior Research, and/or Theory): While obesity and type-2 diabetes rates have risen among Hmong, limited research has investigated their nutritional status. It is important to investigate Hmong dietary and physical activity patterns so that timely nutritional interventions may be planned. Objective: We investigated the factors that influence dietary and physical activity patterns among Hmong American youth (14-25-year-olds) in the Sacramento region.

Study Design, Setting, Participants, Intervention: Eight focus groups $(n=44)$ were conducted. Each focus group lasted for 60-90 minutes with 4 to 7 youth per group. Participants completed a brief questionnaire regarding smoking, alcohol, and dietary and physical activity behaviors adapted from the Centers for Disease Control and Prevention's Youth Risk Surveillance Survey. Focus groups were audio taped and transcribed verbatim; transcriptions were analyzed for themes and discrepancies were noted and resolved.

Outcome Measures and Analysis: Descriptive data were analyzed using IBM SPSS Statistics software (version 24). Significance level was set at $P<.05$.

Results: Twenty-nine female and 15 male youth participated in the study, mean $( \pm S D)$ age was $19 \pm 3$ years. Most youth $(84 \%)$ were born in the United States, seven (16\%) were born in either Laos or Thailand. Questionnaire results indicated that $26 \%$ of youth reported eating fried foods 4-6 times during the past week. Furthermore, 51\% did not eat green salad and 14\% did not consume any fruit during the past week. Focus group data suggested that preference for culturally familiar foods made youth's intake of vegetables challenging. While parental influences negatively influenced female youth's physical activity patterns, cultural expectations to fit in appeared to affect male youth's alcohol consumption.

Conclusions and Implications: Cultural factors affected the youths' health and well-being. Future nutrition intervention among Hmong youth is recommended to be culturally-specific with an understanding of parental influences and/or culturally-driven barriers to healthy living. More data with a larger sample size is needed to gain an understanding about Hmong health in this region.

Funding: Probationary Faculty Development Grant Program at the California State University, Sacramento.

\section{P73 Involving Students and Increasing Communication: Results of a Behavioral Economics Informed Lunchroom Intervention in Middle Schools}

Grace Ryan, MPH, grace-ryan@uiowa.edu, University of Iowa, College of Public Health, N475 College of Public Health Building, 145 N Riverside Drive, Iowa City, IA 52246; Patrick J. Brady, MS; Natoshia M. Askelson, PhD, MPH; Patti Delger, LDN, RD, Iowa Department of Education; Carrie Scheidel, MPH

Objective: The objective of this project was to implement an intervention informed by behavioral economics

Continued on page $S 42$ 